\title{
Impact of maintenance therapy using a half dose of the bacillus Calmette-Guérin Tokyo strain on recurrence of intermediate and high-risk nonmuscle invasive bladder cancer: a retrospective single-center study
}

Dai Koguchi, Kazumasa Matsumoto ${ }^{*}$,Takahiro Hirayama, Shigetaka Moroo, Momoko Kobayashi, Hiroki Katsumata, Masaomi Ikeda and Masatsugu Iwamura

\begin{abstract}
Background: Data are scarce regarding intravesical maintenance therapy (MT) with the low-dose bacillus Calmette-Guérin (BCG) Tokyo strain. We investigated the efficacy and safety of MT with a half dose of the Tokyo strain for patients following transurethral resection of nonmuscle invasive bladder cancer (NMIBC).

Methods: This study retrospectively reviewed clinical data on 78 patients diagnosed with intermediate or high-risk NMIBC followed by either MT $(n=38)$ or IT alone $(n=40)$ between January 2012 and March 2018. Statistical analysis was performed to compare recurrence-free survival (RFS) and adverse effects between the two groups. BCG was instilled once weekly for 6 weeks as IT, then once weekly in 2-week for a total of 20 instillations over 3 years.
\end{abstract}

Results: Kaplan-Meier analyses showed that patients undergoing MT had significantly better RFS than did those undergoing IT alone (hazard ratio (HR):0.32, 95\% confidence interval (Cl):0.12-0.89, $P=0.02$ ). The 3-year RFS was 65.0\% in the IT group and $89.5 \%$ in the MT group. Multivariate analysis showed that MT was associated with a reduced risk of recurrence (HR: 0.32, 95\% Cl:0.11-0.93, $P=0.03)$. One MT patient (2.6\%) exhibited progression.

Conclusions: The BCG Tokyo strain showed acceptable efficacy and safety in patients undergoing MT; thus, it is a potential treatment for preventing bladder cancer recurrence.

Keywords: Bacillus Calmette-Guérin, Bladder cancer, Maintenance therapy, Tokyo strain, Nonmuscle invasive bladder cancer

\section{Background}

Reducing recurrence and preventing progression into muscle invasive disease are crucial issues in managing nonmuscle invasive bladder cancer (NMIBC). According to the European Organization of Research and Treatment

*Correspondence: kazumasa@cd5.so-net.ne.jp

Department of Urology, Kitasato University School of Medicine, 1-15-1

Kitasato, Minami-ku, Sagamihara, Kanagawa 252-0374, Japan of Cancer (EORTC) risk tables [1, 2], intermediate and high-risk NMIBC have high recurrence rates ranging from $24 \%-78 \%$ and a high potential risk of $1 \%-45 \%$ for progressing into muscle invasive disease [3]. Intravesical instillation of bacillus Calmette-Guérin (BCG) is currently the most effective immunotherapy for preventing intravesical recurrence and progression after transurethral resection of intermediate-to-high-risk NMIBC and carcinoma in situ (CIS) $[4,5]$. Although induction 
therapy (IT) followed by maintenance therapy (MT) is recommended for optimal efficacy, BCG-related toxicity remains a major clinical problem, resulting in patients discontinuing the therapy $[6,7]$. The Southwest Oncology Group study (SWOG8507) evidenced that the optimal protocol for instillation maintenance consisted of 3 weekly cycles with 27 instillations over 3 years. However, only $16 \%$ of these patients completed all planned instillations because of severe local and systemic adverse effects (AEs) [8].

Recently, low-dose BCG (LD-BCG) has been shown as a potential option to reduce BCG-associated AEs without affecting the efficacy of full-dose BCG (FD-BCG) for patients following transurethral resection of intermediate-to-high-risk NMIBC [9-11]. For example, a meta-analysis of MT set as 1-yeare period also showed, in comparison with $81 \mathrm{mg}$ the Connaught strain, a significantly better expected overall survival in patients receiving $27 \mathrm{mg}$ with lower probability of AEs [11]. Although such low-dose MT appears promising for treating NMIBC, global BCG shortages due to the sudden cessation of Connaught BCG may limit the MT prevalence. Then, Tokyo strain may be able to overcome the BCG insufficiency because of its' stable supply, and the Food and Drug Administration is testing its efficacy in a new phase III trial in comparison with that of the Tice strain $[13,14]$. However, data are limited regarding the efficacy of MT using the Tokyo strain for LD-BCG, especially with a less intensive regimen than that used in the SWOG $[10,15]$. Previously, a phase II study performed by the Kanagawa Urological Research Group (KURG) showed the potential efficacy of MT with FD-BCG based on a 2-week cycle regimen over 3 years after 6 weeks of IT in patients with intermediate and high-risk NMIBC [16]. Therefore, we retrospectively evaluated the efficacy and safety of MT with a half-dose Tokyo strain following the KURG schedule for those who with intermediate and high-risk NMIBC.

\section{Methods}

\section{Study population}

The study flow chart was illustrated in Fig. 1. The institutional review board at Kitasato University Hospital (B 18-148) approved this study, which was conducted in accordance with the Declaration of Helsinki. This study retrospectively reviewed clinical data on 93 patients diagnosed with intermediate or high-risk NMIBC based on histological examination followed by either MT or IT alone between January 2012 and March 2018. Patients with histories of previous BCG $(\mathrm{n}=15)$ were excluded.

$\mathrm{Ta}$ and $\mathrm{T} 1$ tumors were completely resected via transurethral resection of bladder tumors (TURBT), and cancers were categorized as one of the following, corresponding to intermediate-to-high-risk in the EORTC risk tables (2): (i) presence of at least two bladder tumors, irrespective of primary or recurrent lesion; (ii) single tumor recurring within 12 months of the previous TURBT for NMIBC; or (iii) any high-grade tumor. In cases of CIS, all primary or recurrent biopsy-proven tumors were included, irrespective of concomitant Ta or T1 tumors,

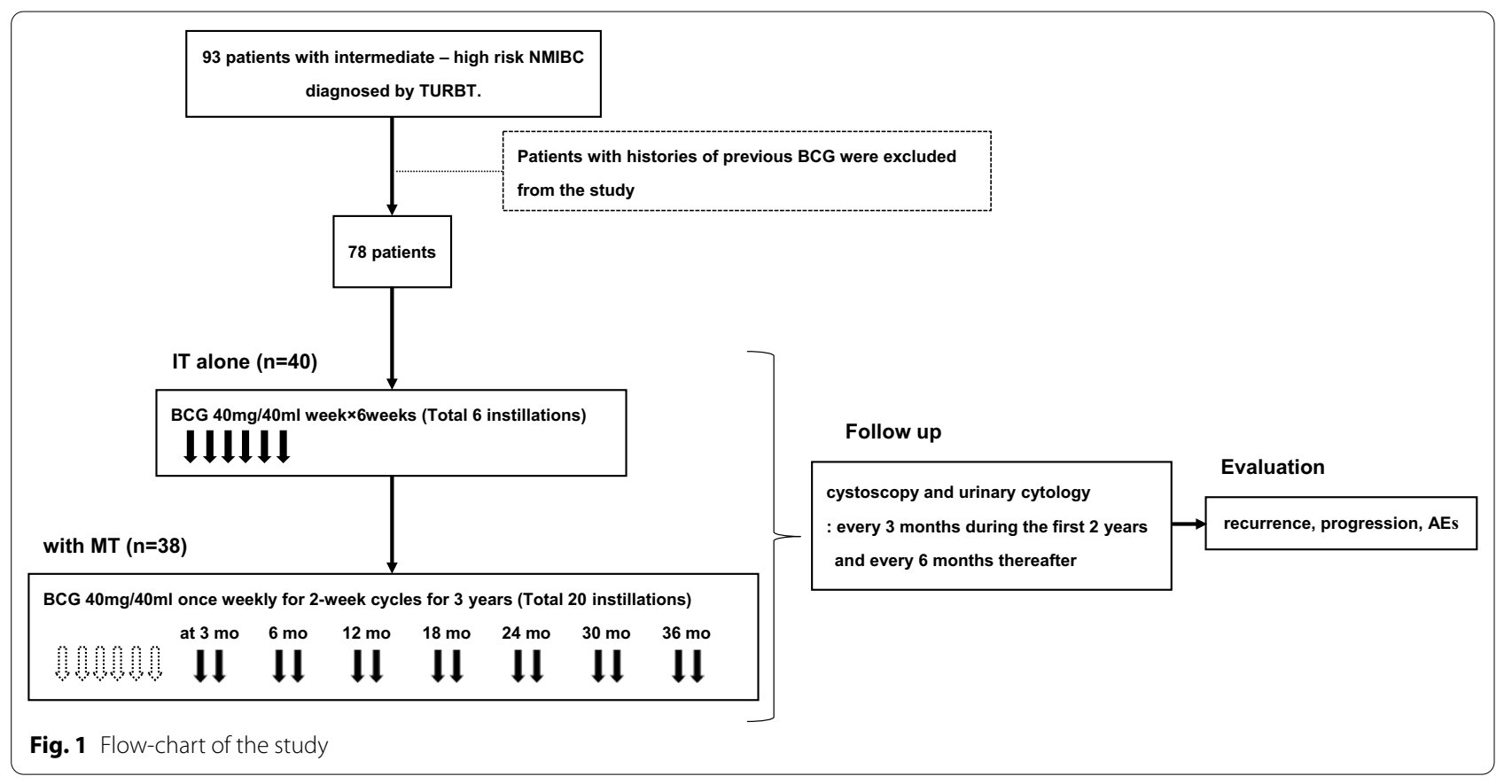


which were completely resected. Exclusion criteria were the presence of lymph nodes or distant metastases, upper tract urothelial or urethral carcinoma, severe infection or any other serious medical complication.

\section{Treatment}

Patients received $40 \mathrm{mg}$ intravesical BCG with the Tokyo strain in $40 \mathrm{ml}$ saline per an instillation during the periods of IT and MT. Treatment method was determined by consultation with the urologist in charge and the patient based on performance status, patient wishes and other factors. BCG solution was instilled into the bladder using a urethral catheter. Patients attempted to retain the solution for $2 \mathrm{~h}$ and then voided. The instillation was started between 7 days and 1 month after TURBT. The instillation was performed once weekly for a total of 6 treatments as IT, then once weekly for 2 -week cycles at months $3,6,12,18,24,30$ and 36 , for a total of 20 instillations over 3 years, as previously reported by the KURG [16].

\section{Study methods}

Treatment efficacy was assessed based on cystoscopy and urinary cytology findings. Cystoscopy and urinary cytology were performed every 3 months during the first 2 years and every 6 months thereafter. BCG instillation was stopped upon recurrence after TURBT, progression to muscle invasive disease, appearance of CIS, positive urinary cytology results, development of carcinoma in the upper urinary tract or prostatic urethra, or distant metastasis. The completion rate was defined as the number of patients with completed instillations during each period (i.e., months $3,6,12,24$ and 36) divided by the total number of eligible patients per group.

The following patient characteristics were collected from the patients' medical charts: age at time of NMIBC, sex, pathological status (i.e., tumor grade and pT stage), tumor size, number of tumors, recurrence rate, time to recurrence from TURBT, progression rate, mortality, AE types and timing, and reasons for discontinuing $\mathrm{MT}$ (categorized as local, systemic AEs and recurrence). AEs were analyzed according to the National Cancer Institute Common Terminology Criteria for Adverse Events, version 4.0. Miction pain, pollakiuria, hematuria, residual urine and urgent urination related to local bladder reactions were categorized as local AEs. Arthralgia, fever of $38.0^{\circ} \mathrm{C}$ or higher and rash were categorized as systemic AEs. Tumor grade was assessed per the 1973 World Health Organization (WHO) grading system. Tumor stage was assessed per the 2002 Tumor Node Metastasis classification of malignant tumors.

\section{Statistical analysis}

Chi-square tests were used to compare the two treatment types to identify relationships between the categorical outcome measures, and Fisher exact test was used when sample sizes is $<10$. Analysis of variance was used for continuous variables. Recurrence-free survival (RFS) was estimated using the Kaplan-Meier method and compared using the log-rank test. Univariate and multivariate analyses were performed to investigate the efficacy of the low-dose MT. All statistical analyses were performed using Stata, ver. 13 for Windows (Stata, Chicago, IL, USA). All $P$-values were two-sided, and $P<0.05$ was considered statistically significant.

\section{Results}

Table 1 lists the patients' characteristics. The study included 61 men (78.2\%) and 17 women (21.8\%) with a median follow-up period of 36.2 months (interquartile range [IQR]:18.2-55.7). Forty patients (51.3\%) received IT alone; 38 (48.7\%) received MT. Patient characteristics did not significantly differ between the two groups.

Overall, 19 patients (24.4\%) had tumor recurrence, with a significantly lower proportion in MT patients than in IT patients (5 patients [13.2\%] vs 14 patients [35.0\%], respectively; $P=0.025)$. Of those with recurrence, seven (36.8\%) experienced recurrence within 6 months after TURBT, and all had undergone IT alone. None of the MT patients showed recurrence before that period $(P=0.046)$. In subgroup analysis of Ta/ 1 group (Ta: $\mathrm{n}=29, \mathrm{~T} 1: \mathrm{n}=31$ ), there was no significant difference in recurrence between patients with pTa and pT1 (7 patients [24.1\%] vs 6 patients [19.4\%], respectively; $P=0.65$ ). In terms of treatment types, no significant difference in recurrence was found between patients with pTa and pT1 (IT: 6 patients [31.6\%] vs 4 patients [28.6\%], respectively, $P=0.85$; MT: 1 patients [10.0\%] vs 2 patients [11.7\%], respectively, $P=0.89$ ).

Kaplan-Meier analyses showed that MT patients had significantly better RFS than did those who underwent IT alone (hazard ratio (HR) 0.32, 95\% confidence interval (CI) $0.12-0.89, P=0.02$; Fig. 2). RFS rates in the IT and MT groups were $70.0 \%$ and $92.1 \%$ at 2 years and $65.0 \%$ and $89.5 \%$ at 3 years, respectively. In the subgroup analysis, no significant differences occurred between patients with Ta/ 1 and Tis (HR $0.31,95 \%$ CI $0.09-1.14, P=0.06$ and HR $0.27,95 \%$ CI $0.50-1.50$, $P=0.11$, respectively; Fig. 3 and 4). Univariate analysis showed that bladder recurrence was associated with BCG treatment type, and multivariate analysis adjusting for the effects of clinicopathological features showed that MT was an independent risk factor for reduced risk of recurrence (Table 2). 
Table 1 Comparison of clinical and pathological characteristics between two treatment types with intravesical bacillus Calmette-Guérin

\begin{tabular}{|c|c|c|c|c|}
\hline & $\begin{array}{l}\text { ALL } \\
(n=78)\end{array}$ & $\begin{array}{l}\text { Induction } \\
(n=40)\end{array}$ & $\begin{array}{l}\text { Maintenance } \\
(n=38)\end{array}$ & $P$ value \\
\hline Age, years (IQR) & $72(64-78)$ & $76(67-79)$ & $76(64-76)$ & 0.36 \\
\hline \multicolumn{5}{|l|}{$\operatorname{Sex}(\%)$} \\
\hline \multirow{2}{*}{$\begin{array}{l}\text { Men } \\
\text { Women }\end{array}$} & $61(78.2)$ & $33(82.5)$ & $28(73.7)$ & \multirow[t]{2}{*}{0.42} \\
\hline & $17(21.8)$ & $7(18.5)$ & $10(26.3)$ & \\
\hline \multicolumn{5}{|l|}{ pT stage (\%) } \\
\hline $\mathrm{pTa} / 1$ & $60(76.9)$ & $33(82.5)$ & $27(71.1)$ & \multirow[t]{2}{*}{0.39} \\
\hline pTis & $18(23.1)$ & $7(18.5)$ & $11(8.9)$ & \\
\hline \multicolumn{5}{|l|}{ Grade (\%) } \\
\hline $\mathrm{G} 1 / 2$ & $63(83.3)$ & $35(87.5)$ & $28(73.7)$ & \multirow[t]{2}{*}{0.16} \\
\hline G3 & $15(16.7)$ & $5(12.5)$ & $10(26.3)$ & \\
\hline \multicolumn{5}{|l|}{ Tumor size (\%) } \\
\hline$<1 \mathrm{~cm}$ & $45(57.7)$ & $25(62.5)$ & $20(52.7)$ & \multirow[t]{2}{*}{0.49} \\
\hline$\geq 1 \mathrm{~cm}$ & $33(42.3)$ & $15(37.5)$ & $18(37.3)$ & \\
\hline \multicolumn{5}{|l|}{ Number of tumors (\%) } \\
\hline Single & $14(17.9)$ & $8(20.0)$ & $6(15.8)$ & \multirow[t]{2}{*}{0.77} \\
\hline Multiple & $64(72.1)$ & $32(80.0)$ & $32(84.2)$ & \\
\hline Follow-up, months (IQR) & $36.2(18.2-55.7)$ & $35.2(19.3-48.3)$ & $35.9(17.0-58.4)$ & 0.25 \\
\hline
\end{tabular}

Unless otherwise stated, values are medians with ranges in parentheses or numbers of patients with percentages in parentheses IQR interquartile range

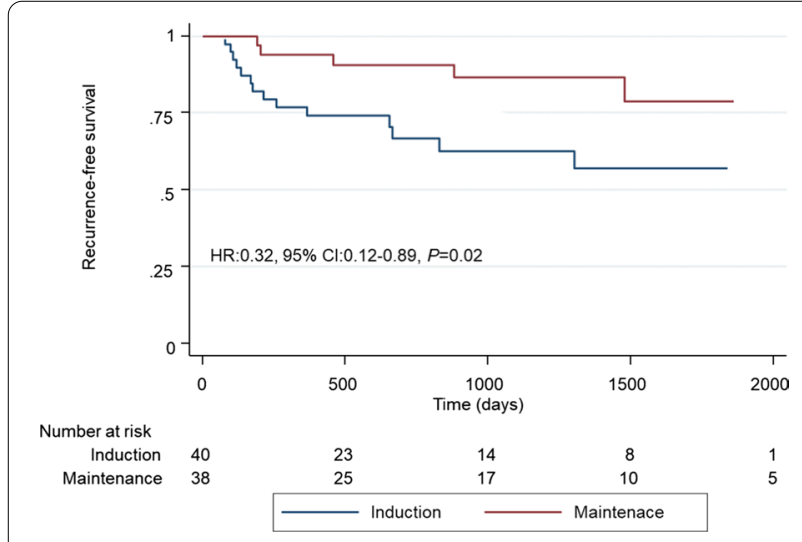

Fig. 2 Kaplan-Meier analysis of recurrence-free survival in all patients

One patient $(2.5 \%)$ in each group experienced tumor progression, with locally advanced progression in the IT patient diagnosed with $\mathrm{pT} 1$ and lymph node metastasis in the MT patient with pTis. Both patients had subsequent intravenous chemotherapy for the progression. Six MT patients $(7.7 \%)$ died: one with tumor progression died of bladder cancer, and the remaining five died of respiratory failure, gallbladder cancer, cervical cancer, esophageal cancer and malignant lymphoma.

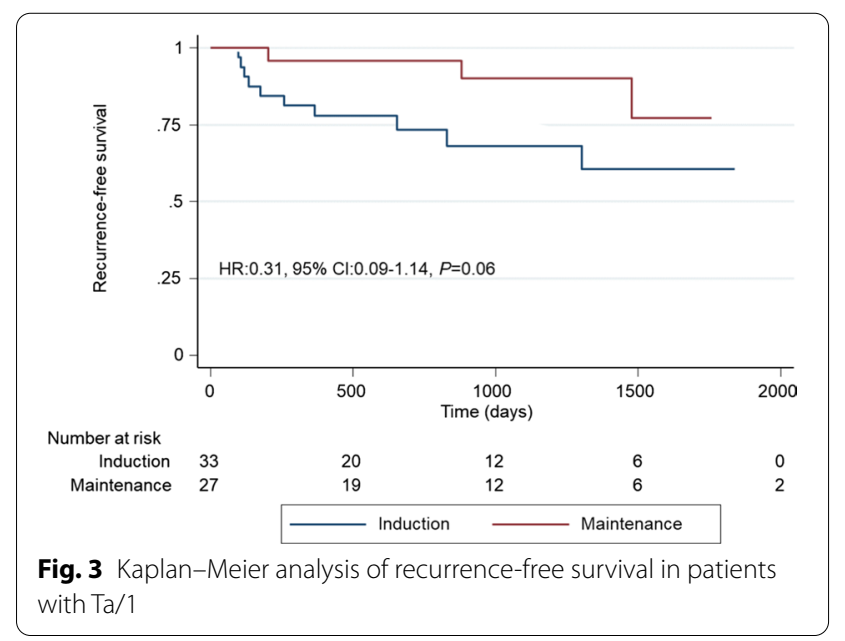

Table 3 lists the AEs. A significantly greater percentage of patients had AEs in the MT group than in the IT group (68.4\% vs. $32.5 \%, P=0.003)$. One patient with IT experienced two systemic AEs: arthralgia and fever. No AEs were classified as $>$ grade 3 in either group. The percentage of patients with AEs during the induction period did not significantly differ between the two groups (MT, 12 patients, $31.6 \%$; IT, 12 patients, $32.5 \% ; P=0.93$ ). In the MT patients, $88.5 \%$ (23/26) experienced AEs until 


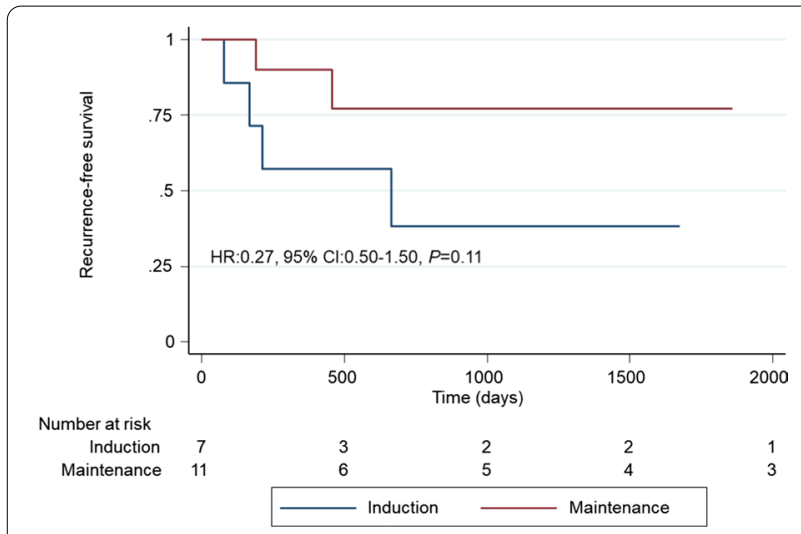

Fig. 4 Kaplan-Meier analysis of recurrence-free survival in patients with Tis

the first 6 months of the maintenance period. Common symptoms were miction pain (36.8\%) and fever (23.7\%).

Maintenance BCG was instilled in 35 patients $(92.1 \%)$ at month 3 , in 27 patients $(71.1 \%)$ at month 6 , in 22 patients $(57.8 \%)$ at month 12 , in 15 patients $(36.8 \%)$ at month 24 , and in 7 patients $(18.4 \%)$ at month 36 . Twentyfour patients stopped MT due to toxicity (12 patients, 31.6\%; 10 local AEs and 2 systemic AEs), recurrence (3 patients, 7.9\%) and others (9 patients, 23.7\%). The remaining seven patients (18.4\%) remained on MT at the time of this analysis.

\section{Discussion}

In this study, MT was performed with a half dose of the Tokyo strain and was continued in 2-week cycles. Data regarding this regimen are extremely limited, even in the era of a BCG shortage [3, 9-11]. We retrospectively investigated the efficacy and safety of MT with LD-BCG for patients following TURBT for intermediate-to-highrisk NMIBC. Patients undergoing MT showed significantly better RFS compared with that of IT alone, and multivariate analysis showed that only MT was associated with a reduced risk of recurrence. Although the overall toxicity with MT was $68.4 \%$, including local and systemic AEs, no AEs were classified as $\geq$ grade 3, and the discontinuation rate of MT due to toxicity was only $31.6 \%$.

Many studies have investigated the feasibility of LDBCG [3, 9]; however, data regarding LD-BCG using the Tokyo strain for patients following TURBT for NMIBC are scarce. With IT, previous cohort studies attained equipotent efficacy at a half dose of the Tokyo strain compared with its standard dose $[17,18]$. Irie et al. reported no significant difference in RFS [17], and a historical cohort study including 156 patients showed no significant differences in RFS or progression-free survival [18]. Only one study analyzed the efficacy of $40 \mathrm{mg}$ of the Tokyo strain as MT [15]. Akaza et al. performed an RCT in which LD-BCG was added to the IT and injected once monthly for a year (totaling 12 instillations), with a 3 -year RFS of $77.8 \%$. In addition to these favorable results of the half dose of the Tokyo strain, both in vitro studies and clinical trials have shown that the Tokyo strain could be an alternative to the Connaught strain thus, the Tokyo strain requires further study, as it may contribute to the stable supply of BCG [19-21].

The widely accepted maintenance protocol for the last 2 decades has been based on SWOG8507. This protocol consists of FD-BCG with 3-week cycles for 3 years,

Table 2 Univariate and multivariate analyses for recurrence-free survival

\begin{tabular}{|c|c|c|c|c|c|c|c|}
\hline \multirow[t]{2}{*}{ Variable } & \multirow[t]{2}{*}{ Category } & \multicolumn{3}{|c|}{ Univariate analysis } & \multicolumn{3}{|c|}{ Multivariate analysis } \\
\hline & & HR & $95 \% \mathrm{Cl}$ & $P$ value & HR & $95 \% \mathrm{Cl}$ & $P$ value \\
\hline \multirow[t]{2}{*}{$\mathrm{BCG}$ treatment } & Maintenance & 0.31 & $0.11-0.88$ & 0.03 & 0.32 & $0.11-0.93$ & 0.03 \\
\hline & Induction & Ref & & & Ref & & \\
\hline Age & & 0.82 & $0.31-2.17$ & 0.70 & 0.58 & $0.20-1.64$ & 0.30 \\
\hline \multirow[t]{2}{*}{ Sex } & Women & 0.73 & $0.43-1.26$ & 0.28 & 1.35 & $0.30-6.13$ & 0.70 \\
\hline & Men & Ref & & & Ref & & \\
\hline \multirow[t]{2}{*}{ Tstage } & $\mathrm{Ta} / 1$ & 1.51 & $082-2.79$ & 0.20 & 0.78 & $0.20-2.95$ & 0.71 \\
\hline & Tis & Ref & & & Ref & & \\
\hline \multirow[t]{2}{*}{ Grade } & $\mathrm{G} 1 / 2$ & 1.39 & $0.70-2.77$ & 0.36 & 1.92 & $0.47-7.80$ & 0.36 \\
\hline & G3 & Ref & & & Ref & & \\
\hline \multirow[t]{2}{*}{ Size } & $<1 \mathrm{~cm}$ & 0.93 & $0.55-1.57$ & 0.79 & 0.38 & $0.13-1.39$ & 0.84 \\
\hline & $\geq 1 \mathrm{~cm}$ & Ref & & & Ref & & \\
\hline \multirow[t]{2}{*}{ Number of tumors } & Single & 0.74 & $0.37-1.48$ & 0.40 & 0.48 & $0.13-1.81$ & 0.28 \\
\hline & Multiple & Ref & & & Ref & & \\
\hline
\end{tabular}


Table 3 Comparison of adverse effects between two treatments types with intravesical bacillus Calmette-Guérin

\begin{tabular}{|c|c|c|c|c|}
\hline & $\operatorname{ALL}(n=78)$ & Induction $(n=40)$ & Maintenance $(n=38)$ & $P$ value \\
\hline \multicolumn{5}{|l|}{ Adverse effects (\%) } \\
\hline Grade $\leq 2 /$ Grade $\geq 3$ & $39(50.0) / 0$ & $13(32.5) / 0$ & $26(68.4) / 0$ & 0.003 \\
\hline Local & $30(38.6)$ & $9(22.5)$ & $21(57.9)$ & 0.003 \\
\hline Systemic & $16(20.5)$ & $7(17.5)$ & $9(23.7)$ & 0.50 \\
\hline Both & $7(9.0)$ & $3(7.5)$ & $4(10.5)$ & 0.64 \\
\hline \multicolumn{5}{|l|}{ Local symptoms (\%) } \\
\hline Miction pain & $18(23.1)$ & $4(10.0)$ & $14(36.8)$ & $<0.001$ \\
\hline Pollakiuria & $8(10.3)$ & $3(7.5)$ & $5(13.2)$ & 0.41 \\
\hline Hematuria & $6(7.7)$ & $1(2.5)$ & $5(13.2)$ & 0.10 \\
\hline Residual urine & $2(2.6)$ & 0 & $2(5.3)$ & 0.23 \\
\hline Urgent urination & $1(1.3)$ & $1(2.5)$ & 0 & 0.33 \\
\hline \multicolumn{5}{|l|}{ Systemic symptoms (\%) } \\
\hline Arthralgia & $1(1.3)$ & $1(2.5)$ & 0 & 0.33 \\
\hline Fever & $15(19.2)$ & $6(15.0)$ & $9(23.7)$ & 0.40 \\
\hline Rash & $1(1.3)$ & $1(2.5)$ & 0 & 0.33 \\
\hline
\end{tabular}

Unless otherwise stated, values are numbers of patients with percentages in parentheses

totaling 27 instillations. However, recent evidence suggests that the SWOG regimen may include unnecessary BCG instillations [3, 9]. A meta-analysis recently indicated a nonlinear association between the dose per instillation and clinical efficacy [9]. A large phase III RCT (EORTC30962) found that the 5-year RFS rates were $54.5 \%, 58.8 \%, 62.6 \%$, and $64.2 \%$ for $1 / 3$-dose/ 1 year, full-dose/ 1 year, $1 / 3$-dose/3 years, and full-dose/3 years, respectively $(P=0.038)$ [22]. Compared with a large RCT based on SWOG8507, the present study with 2-week cycles for 3 years with 20 instillations in total showed acceptable RFS. The 2-year RFS was $92.1 \%$, and the 3 -year RFS was $89.1 \%$ in the present study, while the 2-year RFS was $82 \%$ in SWOG8507, and the 3-year RFS was $65.0 \%$ in EORTC30911 [8, 23]. The KURG study supported the potential efficacy of the 2 -week cycle regimen. This prospective phase II study of MT with FD-BCG followed the same instillation schedule as ours, showing a 3-year RFS of about $80 \%$ [16]. To date, although controversies surround the efficacy of MT with LD-BCG, the American Urological Association suggested reducing the BCG dose from an economical point of view [24-26]. Considering the recurrence, progression rate, and toxicity, the present study implied a favorable cost-effectiveness of MT using a half dose compared with the FD-BCG. Further large prospective studies are needed to find an optimal reducedintensity regimen in terms of dose and frequency using various BCG strains.

Previous studies have evidenced positive efficacy with the 2-week cycle [27-29]. A prospective study found that high urinary leukocytes predicted MT efficacy in patients with NMIBC and a level of urinary leukocytosis and Th1 peaked at around the second instillation. [27, 28]. A recent analysis revealed increased blood platelets after 2-4 BCG instillations in patients with NMIBC. These instillations may introduce leukocyte infiltration into the stromal tissue and modulate the effector functions of neutrophils and macrophages [29]. Although the optimal MT schedule is unclear $[3,9,11]$, these patterns of immunological responses to intravesical BCG instillations reinforced the clinical outcomes of the present study based on the 2-week cycle.

LD-BCG has been used in efforts to reduce AEs and secondarily improve MT completion rates [9, 10, 30]. Nevertheless, compared with previous studies using FDBCG for the same 3-year period, the present study with the half-dose Tokyo strain showed a similar toxicity rate with a lower completion rate. Local and systemic AE rates were $57.9 \%$ and $23.7 \%$ in the present study, $63.3 \%$ and $33.6 \%$ in the EORTC30962, and $67.5 \%$ and $22.5 \%$ in a recent large retrospective study in Japan, respectively $[31,32]$. The completion rate was $18.4 \%$ in the present study and 35\% in both KURG and EORTC3092 [16, 31]. However, in the present study, no patients had AEs above grade 3, compared with about $10 \%$ in the KURG study [16]. Furthermore, the main reasons for discontinuation were unrelated to physical AEs. These reasons included drop-out and patients' preferences, which accounted for 23.7\%. Hence, physical AEs may not always accurately reflect the MT discontinuation [33]; this is supported by accumulating evidence showing that most AEs during MT occur within the first year, then decrease gradually thereafter in inverse proportion to the number of patients discontinuing MT [31, 32]. Therefore, further 
analyses of toxicity, including emotional aspects such as patients' quality of life, should be analyzed to increase the completion rate.

This study had some limitations. First, the study was retrospective, and the association between previously collected data and clinical outcomes in the current study may result from unmeasured or residual confounding by other factors. Second, the WHO 1973 classification might require a careful understanding of the diagnosis of particularly Grade 2 diseases. Third, statistical analyses of clinical outcomes other than RFS were insufficient; however, only one patient per group progressed to bladder carcinoma, and only one MT patient died of bladder carcinoma. A longer follow-up may enable performing appropriate statistical analyses of such events with larger numbers. Forth, seven patients remained on MT at the time of this analysis; four of seven patients had completed over 2 years of follow-up, and two of the remaining three patients completed over 1 year of follow-up. Thus, we believe that the follow-up periods were sufficient to evaluate the clinical outcomes.

\section{Conclusions}

The present study using the half-dose Tokyo strain and 2-week cycles showed acceptable clinical outcomes with no AEs classified above grade 3 in the MT group. Furthermore, multivariate analysis showed that MT was an independent reduced risk factor of recurrence. Therefore, the present reduced-intensity regimen relative to that of the SWOG should be strongly considered during a BCG shortage and should be validated in further large prospective studies that also evaluate patients' emotional aspects for better managing maintenance periods.

\section{Abbreviations \\ AEs: Adverse effects; BCG: Bacillus Calmette-Guérin; CIS: Carcinoma in situ; CUETO: Club Urológico Español de Tratamiento Oncológico; Cl: Confidence interval; EORTC: European Organization of Research and Treatment of Cancer; FD-BCG: Full-dose BCG; HR: Hazard ratio; IT: Induction therapy; KURG : Kanagawa Urological Research Group; LD-BCG: Low-dose BCG; NMIBC: Non- muscle invasive bladder cancer; MT: Maintenance therapy; RCTs: Randomized control trials; RFS: Recurrence-free survival; SWOG: Southwest Oncology Group; TURBT: Transurethral resection of bladder tumors; WHO: World Health Organization.}

\section{Acknowledgements}

Not applicable.

\section{Authors' contributions}

DK: Manuscript writing, Data analysis; KM: Manuscript editing, Project development, Data analysis and collection; TH: Project development, Data analysis and collection; SM: Data collection; MK: Data collection; HK: Data collection; MI1: Data analysis and collection; MI2: Project development. All authors read and approved the final manuscript.

\section{Funding}

This research did not receive any specific grant from any funding agency.

\section{Availability of data and materials}

The datasets used and/or analysed during the current study are available from the corresponding author on reasonable request.

\section{Ethics approval and consent to participate}

The institutional review board at Kitasato University Hospital (B 18-148) approved this study, including the request to waive the documentation of informed consent.

\section{Consent for publication \\ Not applicable.}

\section{Competing interests}

The authors declare that they have no competing interests.

Received: 2 July 2020 Accepted: 1 December 2020

Published online: 09 December 2020

\section{References}

1. Borkowska EM, Jedrzejczyk A, Marks P, Catto JWF, Kaluzewski B. EORTC risk tables - their usefulness in the assessment of recurrence and progression risk in nonmuscle-invasive bladder cancer in Polish patients. Cent Eur J Urol. 2013;66(1):14-20.

2. Walczak R, Bar K, Walczak J. The value of EORTC risk tables in evaluating recurrent non-muscle-invasive bladder cancer in everyday practice. Cent Eur J Urol. 2014;66(4):418-22.

3. Quan Y, Jeong CW, Kwak C, Kim HH, Kim HS, Ku JH. Dose, duration and strain of Bacillus Calmette-Guerin in the treatment of nonmuscle invasive bladder cancer: meta-analysis of randomized clinical trials. Medicine. 2017;96(42):e8300.

4. Power NE, Izawa J. Comparison of guidelines on non-muscle invasive bladder cancer (EAU, CUA, AUA, NCCN, NICE). Bladder Cancer. 2016;2(1):27-36.

5. Morales A, Eidinger D, Bruce AW. Intracavitary Bacillus Calmette-Guerin in the treatment of superficial bladder tumors. J Urol. 1976;116(2):180-3.

6. European Association of Urology. EAU Guidelines on non-muscle-invasive bladder cancer (TaT1). 2017. https://uroweb.org/wp-content/uploads/ EAU-Guidelines-on-Non-muscle-Invasive-BC_TaT1-2017. Accessed 30 May 2020.

7. Spiess PE, Agarwal N, Bangs R, Boorjian SA, Buyyounouski MK, Clark PE, et al. NCCN clinical practice guidelines in oncology. J Natl Compr Canc Netw. 2017;15(10):1240-67.

8. Lamm DL, Blumenstein BA, Crissman JD, Montie JE, Gottesman JE, Lowe BA, et al. Maintenance Bacillus Calmette-Guerin immunotherapy for recurrent TA, T1 and carcinoma in situ transitional cell carcinoma of the bladder: a randomized Southwest Oncology Group Study. J Urol. 2000;163(4):1124-9.

9. Wu C, Zhou X, Miao C, Zhang J, Tang Q, Chang X, et al. Assessing the feasibility of replacing standard-dose bacillus Calmette-Guérin immunotherapy with other intravesical instillation therapies in bladder cancer patients: a network meta-analysis. Cell Physiol Biochem. 2017:41(4):1298-312.

10. Zeng S, Yu X, Ma C, Zhang Z, Song R, Chen X, et al. Low-dose versus standard dose of Bacillus Calmette-Guerin in the treatment of nonmuscle invasive bladder cancer: a systematic review and meta-analysis. Medicine. 2015;94(49):e2176.

11. Wang Z, Xiao H, Wei G, Zhang N, Wei M, Chen Z, et al. Low-dose Bacillus Calmette-Guerin versus full-dose for intermediate and high-risk of non-muscle invasive bladder cancer: a Markov model. BMC Cancer. 2018;18(1):1108.

12. Miyazaki J, Onozawa M, Takaoka E, Yano I. Bacillus Calmette-Guérin strain differences as the basis for immunotherapies against bladder cancer. Int J Urol. 2018;25(5):405-13.

13. Cernuschi T, Malvolti S, Nickels E, Friede M. Bacillus Calmette-Guérin (BCG) vaccine: a global assessment of demand and supply balance. Vaccine. 2018;36(4):498-506.

14. Svatek RS, Tangen C, Delacroix S, Lowrance W, Lerner SP. Background and update for S1602 "a phase III randomized trial to evaluate the influ- 
ence of BCG strain differences and T cell priming with intradermal BCG before intravesical therapy for BCG-naive high-grade non-muscle invasive bladder cancer. Eur Urol Focus. 2018;4(4):522-4.

15. Akaza H, Hinotsu S, Aso Y, Kakizoe T, Koiso K. Bacillus Calmette-Guérin treatment of existing papillary bladder cancer and carcinoma in situ of the bladder. Four-year results. The Bladder Cancer BCG Study Group. Cancer. 1995;75(2):552-9.

16. Kanagawa Urological Research Group (KURG). A 2-week maintenance regimen of intravesical instillation of Bacillus Calmette-Guerin is safe, adherent and effective in patients with non-muscle-invasive bladder cancer: a prospective, multicenter phase II clinical trial. Jpn J Clin Oncol. 2012;42(9):813-9.

17. Irie A, Uchida T, Yamashita H, Matsumoto K, Satoh T, Koh H, et al. Sufficient prophylactic efficacy with minor adverse effects by intravesical instillation of low-dose Bacillus Calmette-Guérin for superficial bladder cancer recurrence. Int J Urol. 2003;10(4):183-9.

18. Yoneyama T, Ohyama C, Imai A, Ishimura H, Hagisawa S, Iwabuchi I, et al. Low-dose instillation therapy with Bacille Calmette-Guérin Tokyo 172 strain after transurethral resection: historical cohort study. Urology. 2008;71(6):1161-5.

19. Ikeda N, Honda I, Yano I, Koyama A, Toida I. Bacillus calmette-guerin Tokyo 172 substrain for superficial bladder cancer: characterization and antitumor effect. J Urol. 2005;173(5):1507-12.

20. Boehm BE, Cornell JE, Wang H, Mukherjee N, Oppenheimer JS, Svatek RS. Efficacy of bacillus Calmette-Guérin strains for treatment of nonmuscle invasive bladder cancer: a systematic review and network meta-analysis. J Urol. 2017;198(3):503-10.

21. Sengiku A, Ito M, Miyazaki Y, Sawazaki H, Takahashi T, Ogura K. A prospective comparative study of intravesical Bacillus Calmette-Guérin therapy with the Tokyo or Connaught strain for nonmuscle invasive bladder cancer. J Urol. 2013:190(1):50-4.

22. Oddens JR, Brausi M, Sylvester RJ, Bono A, van de Beek C, van Andel G, et al. Final results of an EORTCGU cancers group randomized study of maintenance Bacillus Calmette-Guérin in intermediate- and highrisk Ta, T1 papillary carcinoma of the urinary bladder: one-third dose versus full dose and 1 year versus 3 years of maintenance. Eur Urol. 2013;63(3):462-72.

23. van der Meijden AP, Brausi M, Zambon V, Kirkels W, de Balincourt C, Sylvester R, et al. Intravesical instillation of epirubicin, Bacillus CalmetteGuérin and Bacillus Calmette-Guérin plus isoniazid for intermediate and high risk $\mathrm{Ta}, \mathrm{T} 1$ papillary carcinoma of the bladder: a European Organization for Research and Treatment of Cancer genito-urinary group randomized phase III trial. J Uro. 2001;166(2):476-81.

24. Martínez-Piñeiro L, Portillo JA, Fernández JM, Zabala JA, Cadierno I, Moyano JL, et al. Maintenance Therapy with 3-monthly Bacillus
Calmette-Guérin for 3 Years is Not Superior to Standard Induction Therapy in High-risk Non-muscle-invasive Urothelial Bladder Carcinoma: Final Results of Randomised CUETO Study 98013. Eur Urol. 2015;68(2):256-62.

25. American Urological Association. AUA Practice Resources: BCG Shortage Notice. 2019. https://www.auanet.org/practice-resources/bcg-info/bcgshortage-notice. Accessed 20 May 2020.

26. Sharma V, Wymer KM, Borah BJ, Saigal CS, Litwin MS, Packiam VT, et al. Cost-effectiveness of maintenance BCG for intermediate and high risk non-muscle invasive bladder cancer. J Urol. 2020. https://doi.org/10.1097/ JU.0000000000001023.

27. Saint F, Patard JJ, Irani J, Salomon L, Hoznek A, Legrand P, et al. Leukocyturia as a predictor of tolerance and efficacy of intravesical BCG maintenance therapy for superficial bladder cancer. Urology. 2001;57(4):617-21.

28. de Reijke TM, Boer ECDE, Kurth KH, Schamhart DH. Urinary interleukin-2 monitoring during prolonged Bacillus Calmette- Guerin treatment: Can it predict the optimal number of instillations? J Urol. 1999;161(1):67-71.

29. Miyake M, Hori S, Ohnishi S, Owari T, lida K, Ohnishi K, et al. Clinical impact of the increase in immunosuppressive cell-related gene expression in urine sediment during intravesical Bacillus Calmette-Guérin. Diseases. 2019;7(2):44.

30. Martínez-Piñeiro JA, Flores N, Isorna S, Solsona E, Sebastián JL, Pertusa C, et al. Long-term follow-up of a randomized prospective trial comparing a standard $81 \mathrm{mg}$ dose of intravesical Bacille Calmette-Guérin with a reduced dose of $27 \mathrm{mg}$ in superficial bladder cancer. BJU Int. 2002;89(7):671-80.

31. Brausi M, Oddens J, Sylvester R, Bono A, van de Beek C, van Andel G, et al. Side effects of Bacillus Calmette-Guérin (BCG) in the treatment of intermediate- and high-risk Ta, T1 papillary carcinoma of the bladder: results of the EORTC genito-urinary cancers group randomised Phase 3 study comparing one-third dose with full dose and 1 year with 3 years of maintenance BCG. Eur Assoc Urol. 2014;65(1):69-76.

32. Muto S, Nakajima A, Horiuchi A, Inoue M, China T, Saito K, et al. Maintenance therapy with intravesical bacillus Calmette-Guérin in patients with intermediate- or high-risk non-muscle-invasive bladder cancer. Jpn J Clin Oncol. 2013;43(3):305-13.

33. Russell JS, Colevas AD. Adverse event monitoring in oncology clinical trials. Clin Invest. 2013;3(12):1157-65.

\section{Publisher's Note}

Springer Nature remains neutral with regard to jurisdictional claims in published maps and institutional affiliations.
Ready to submit your research? Choose BMC and benefit from:

- fast, convenient online submission

- thorough peer review by experienced researchers in your field

- rapid publication on acceptance

- support for research data, including large and complex data types

- gold Open Access which fosters wider collaboration and increased citations

- maximum visibility for your research: over $100 \mathrm{M}$ website views per year

At BMC, research is always in progress.

Learn more biomedcentral.com/submissions 